\title{
Laparoscopic Repair of Diaphragmatic Hernia after Left Ventricular Assist Device Explantation
}

\author{
Amy Liang, MD, Michael Passeri, MD, Grace J. Kim, MD \\ Department of Surgery, Mt. Sinai-St. Luke's-Roosevelt Hospital Center, New York, New York, USA (all authors).
}

\begin{abstract}
Introduction: Herniation of the bowel through a diaphragmatic defect is a rare cause of abdominal pain and obstruction in the adult patient. Most clinically significant congenital hernias are discovered in childhood, and most acquired traumatic and iatrogenic defects are discovered shortly after the injury, if at all. Delayed symptomatic herniation years after initial insult is an uncommon but well-described complication after diaphragmatic injury.

Case Description: A 34-year-old man presented to the emergency department 10 hours after developing sharp left upper quadrant abdominal pain, accompanied by nausea and dry heaves. His surgical history was significant for sequential left ventricular assist device explantation and orthotopic heart transplantation 2 years prior. A chest radiograph demonstrated a loop of colon in the left chest. The patient was taken to the operating room for emergent laparoscopy, reduction, and repair of an incarcerated diaphragmatic hernia. A laparoscopic approach was used to reduce and evaluate the viscera. The hernia defect, which measured $13 \mathrm{~cm}^{2}$, was then closed primarily with nonabsorbable sutures. The patient was discharged uneventfully on postoperative day 2 .
\end{abstract}

Discussion: Although uncommon, left ventricular assist device placement and explantation can lead to iatrogenic diaphragmatic hernia. The possibility of a diaphragmatic hernia should be considered in any patient presenting with abdominal pain after left ventricular assist device explantation. A laparoscopic repair should be used when possible.

Key Words: Left Ventricular Assist Device, Diaphragmatic Hernia, Heart Transplant

Citation Liang A, Passeri M, Kim GJ. Laparoscopic repair of diaphragmatic hernia after left ventricular assist device explantation. CRSLS e2016.00042. DOI: 10.4293/CRSLS.2016.00042.

Copyright $(0) 2016$ by SLS, Society of Laparoendoscopic Surgeons. This is an open-access article distributed under the terms of the Creative Commons Attribution-Noncommercial-ShareAlike 3.0 Unported license, which permits unrestricted noncommercial use, distribution, and reproduction in any medium, provided the original author and source are credited.

Address correspondence to: Michael Passeri, MD, Department of Surgery, Mt. Sinai St. Luke's-Roosevelt Hospital Center, 1000 Tenth Avenue, Surgery Offices, Second Floor, NY, NY 10019. Telephone: 212-523-6970, Fax: 212-523-6495, E-mail: Mpasseri@chpnet.org

\section{INTRODUCTION}

Symptomatic herniation of abdominal contents through a nonhiatal diaphragmatic defect is a rare diagnosis in the adult patient. Most reported cases can be categorized into 1 of 2 scenarios: the adult presentation of a congenital diaphragmatic hernia or herniation after diaphragmatic injury.

The true incidence of abdominal organ herniation after thoracoabdominal injury is unclear, because many hernias are thought to be asymptomatic. ${ }^{1}$ It is not unusual for traumatic hernias to be discovered incidentally on imaging studies years after injury. They may also present with symptoms including abdominal pain, nausea, vomiting, chest discomfort, and coughing.
Regarding iatrogenic diaphragmatic injury, numerous mechanisms have been described. A particularly rare example is injury during sequential left ventricular assist device (LVAD) explantation and orthotopic heart transplantation (OHT).

LVADs provide cardiovascular support as a bridge to OHT, and placements have been increasing in the United States. ${ }^{2,3}$ In LVAD placement, the diaphragm is penetrated in 2 places for the inflow and outflow cannulae, with potential development of diaphragmatic herniation in either location after LVAD explantation. ${ }^{4,5}$ In total, we found 17 cases of diaphragmatic hernia after LVAD explantation reported in the literature. ${ }^{5-10}$ Time to presentation after OHT ranges from 6 months to 8.5 years. ${ }^{5-8}$ Data regarding the presentation, diagnosis, and treatment are limited. 
The identification of a nonhiatal diaphragmatic defect of any etiology is an indication for operative repair. In the era of minimally invasive surgery, both thoracoscopic and laparoscopic approaches have been described. Neither approach has demonstrated clear superiority, though each may have advantages. Thoracoscopy is often preferred when the hernia sac is suspected to be densely adherent to other structures in the chest, whereas laparoscopy has the advantage of avoiding double-lumen intubation and enhances the ability to inspect the herniated viscera for viability.

We describe a case of diaphragmatic herniation that was diagnosed 2 years after LVAD explanation and OHT. Our patient presented to the emergency department with abdominal pain and symptoms of obstruction. A primary laparoscopic repair was performed.

\section{CASE REPORT}

A 34-year-old man presented to our emergency department with the chief concern of left upper quadrant (LUQ) abdominal pain that had started $\sim 10$ hours before arrival. The pain was described as sharp, severe, and accompanied by nausea and dry heaves without vomiting. He denied any history of trauma to chest or abdomen. He reported a history of heart failure secondary to viral cardiomyopathy that had been managed by LVAD placement, followed by heart transplantation 2 years earlier. At the time of presentation, he was taking tacrolimus and mycophenolate for immunosuppression.

On examination, the patient was seen resting in bed in moderate discomfort. He had normal bowel sounds and a soft, nondistended abdomen. Palpation demonstrated LUQ tenderness without guarding or rebound tenderness. There were no palpable masses or hernias. His sternotomy incision was well-healed, and his breath sounds were clear. Laboratory values including electrolytes, lactate, $\mathrm{CO}_{2}$, and white blood cell (WBC) count were unremarkable.

A chest $\mathrm{x}$-ray demonstrated a loop of colon herniating into the left hemithorax (Figure 1). The diagnosis was an incarcerated diaphragmatic hernia, presumably iatrogenic secondary to prior cardiac surgery, and the decision was made to take the patient to the operating room for emergent repair.

The patient was placed supine on the operating table with arms tucked. A Veress needle was used to access the abdomen at the umbilicus, and pneumoperitoneum was achieved. The Veress needle was exchanged for a $5-\mathrm{mm}$ optical trocar. Additional trocars were placed under direct

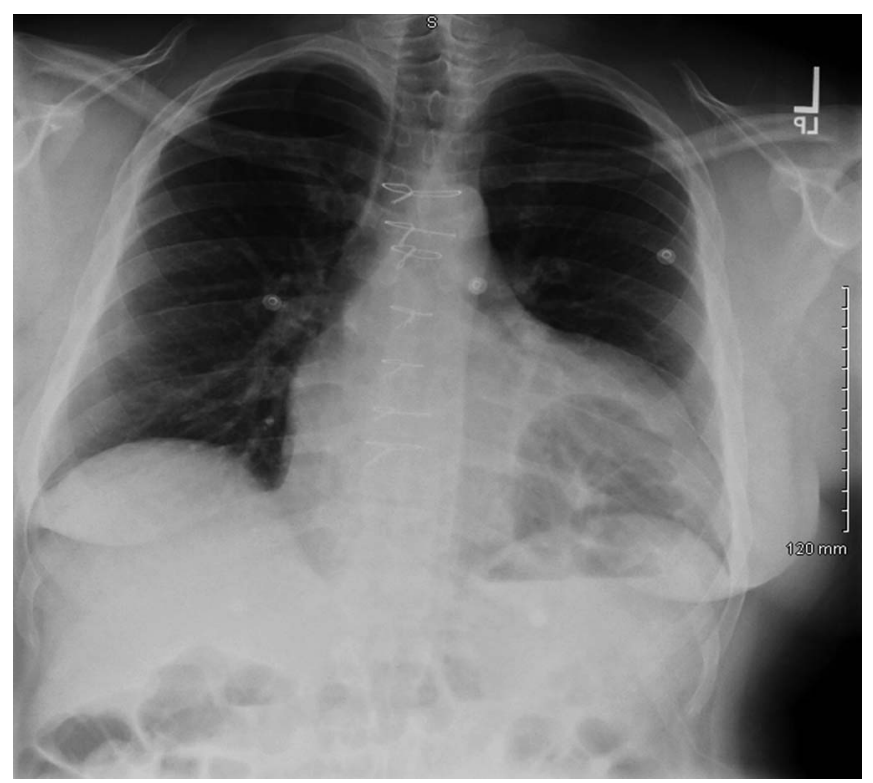

Figure 1. Chest $x$-ray demonstrating the bowel herniating into the left hemithorax.

laparoscopic vision: a 5-mm trocar in the midclavicular line in the right subcostal space, a $5-\mathrm{mm}$ trocar in the midclavicular line in the left subcostal space, and a $12-\mathrm{mm}$ trocar in the left anterior axillary line. The diaphragm was visualized, and the omentum and transverse colon were seen herniated within the defect (Figure 2).

As the herniated contents were reduced back into the abdomen, several points of adhesion to the thoracic cavity were noted. These were divided with the ultrasonic shears. The reduced transverse colon was inspected and determined to be viable. Attention was then turned to the diaphragmatic hernia, which was a clearly defined circular

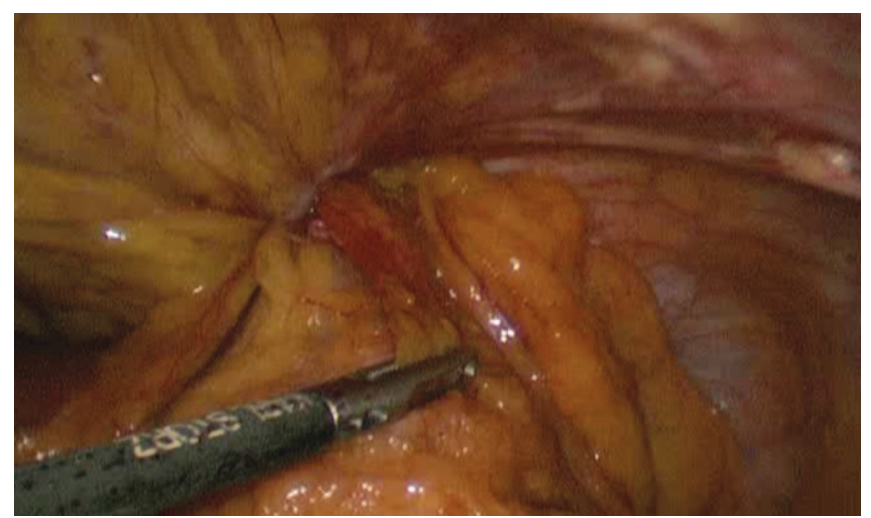

Figure 2. Laparoscopic view of omental fat herniating up through the left-side diaphragmatic defect. Herniated transverse colon, also present, is obscured by the omental fat. 
defect with an area of $13 \mathrm{~cm}^{2}$ (Figure 3). A primary closure using interrupted, 0 simple sutures (Ethibond; Ethicon, Somerville, New Jersey, USA) on a curved needle was then performed from the edges toward the center (Figure 4).

After the operation, the patient made an uneventful recovery. A chest x-ray in the recovery room demonstrated complete reduction of the bowel loops and reinflation of the left lung. The patient's diet was advanced and he was discharged home two days after his operation on a soft diet. At his follow-up office visit after 4 weeks, he was doing well.

\section{DISCUSSION}

Prior abdominal or thoracic surgery can lead to cases of traumatic diaphragmatic herniation. A particularly uncommon iatrogenic diaphragmatic hernia is one that occurs after LVAD placement and explantation, as described in this case report.

In LVAD placement, the apex of the left ventricle is used to establish inflow to the pump, and the aorta is used as the outflow to the aorta, thereby bypassing the failing left ventricle.,3 The pump is typically placed in an intraperitoneal, preperitoneal, or intramuscular pocket in the abdomen, and catheters are tunneled into the left pleural space. The diaphragm is penetrated in 2 distinct locations, by outflow and inflow catheters, resulting in the possibility of the development of 2 distinct hernias. A left transdiaphragmatic hernia would result from the inflow cannula to the left ventricle, and an anterior defect would result from the outflow cannula from the aorta. ${ }^{6}$ It was suspected that the diaphragmatic hernia that was repaired in this patient was from the inflow cannula, based on its location.

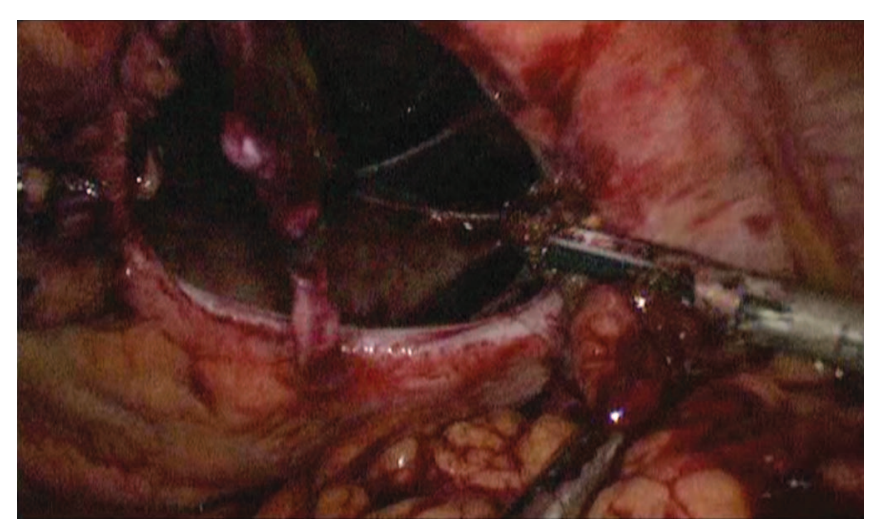

Figure 3. View from the abdomen up through the diaphragmatic defect into the left hemithorax.

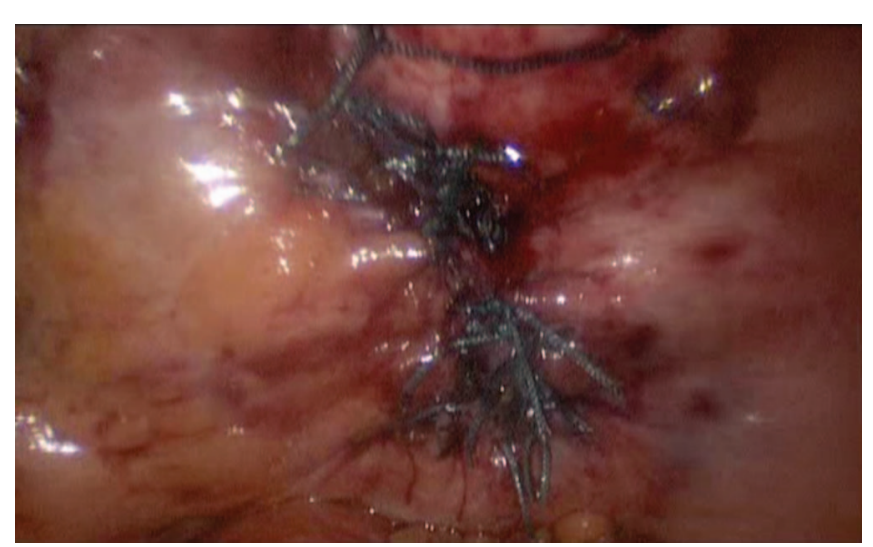

Figure 4. View of the primarily repaired diaphragmatic defect, which was closed with interrupted 0 sutures.

The first report of diaphragmatic hernia following LVAD explantation was in 1992. ${ }^{5}$ Since then, 17 cases have been described in the literature, and of those, 14 were repairs. ${ }^{5-10}$ Several technical variations in the placement and removal of LVADs that may predispose to diaphragmatic hernia have been studied. Intraperitoneal placement has been shown to carry a higher risk of diaphragmatic hernia compared to the extraperitoneal approach. In addition, according to one small series, if the cannula defects are left open at the time of explantation, the reported incidence of hernia is $15.9 \%$; if closed, the incidence is $4.3 \%{ }^{6}$ A subsequent series in which all defects were closed reported an incidence of $5.2 \% .^{10}$

The possibility of a diaphragmatic hernia must be considered in any patient who presents with abdominal pain after prior LVAD placement and explantation. Because of the unique aspects of this patient population including immunosuppression, multiple prior surgeries and history of heart failure, a diaphragmatic hernia may readily evolve into a life-threatening event, and the possibility of a diaphragmatic hernia must be investigated, and expeditious surgical care must be implemented.

As with most diaphragmatic hernias, these hernias may be approached through the chest or abdomen. However, surgeons who favor the abdominal approach have noted that unlike the traumatic diaphragmatic hernia population, all patients in the LVAD setting would have had at least 2 prior median sternotomies, resulting in a chest more extensively scarred than the abdomen. Even in the cases where the LVAD had been positioned intraperitoneally and a hostile abdomen may be anticipated, the abdominal approach may be preferred, as the bowel adhesions are likely to be more safely approached intraabdominally. ${ }^{6}$ 
Since the report of laparoscopic repair of diaphragmatic hernia after LVAD explantation by Farma et al in 2003, the minimally invasive approach has become the standard of care in the treatment of these types of hernias when technically possible.9,10 Consistent with other laparoscopic applications, numerous reports have shown a decreased length of stay without increased recurrence rate in the approach to these types of hernias. ${ }^{11,12}$ Moreover, the basic benefits of laparoscopy including better visualization of the diaphragm, less pulmonary embarrassment, earlier resumption of oral intake, and lower wound infection rate ${ }^{13,14}$ would perhaps be amplified in this uniquely fragile and immunosuppressed patient population with history of heart failure and multiple prior chest operations. This report examines only a single case and follow-up is limited, but the case seems uniquely suited for the application of the laparoscopic approach if technically achievable.

As the frequency of LVAD placement continues to rise in the United States and with improving survival after cardiac transplantation, it is likely that the incidence of diaphragmatic hernias after LVAD will continue to increase..$^{2,3}$ The possibility of a diaphragmatic hernia should be considered in any patient presenting with abdominal pain after LVAD. Because of the uniquely debilitated nature of this patient population, such a complication carries potentially higher morbidity and mortality, and the possibility of this complication should be vigorously investigated and treated.

\section{CONCLUSION}

Diaphragmatic trauma secondary to LVAD placement is likely to become more common as the incidence of LVAD placement increases. The possibility of a diaphragmatic hernia must be considered in any patient with a history of LVAD placement who presents with abdominal pain or symptoms of obstruction. The laparoscopic approach has obvious benefits that are intuitively amplified in this particular patient population. More study is needed to determine what measures can be done at the time of LVAD explanation to minimize the possibility of this complication.

\section{References:}

1. Liao $\mathrm{CH}$, Chu $\mathrm{CH}$, Wu YT, et al. The feasibility and efficacy of laparoscopic repair of chronic traumatic diaphragmatic her- niation: introduction of a novel technique with literature review. Hernia. 2016;20:303-309.

2. McCarthy FH, Kobrin D, Rame JE, et al. Increasing frequency of left ventricular assist device exchanges in the United States. Ann Thorac Surg. 2015;100:1660-1664; discussion 1665 .

3. Kirklin JK, Pambukian SV, McGiffin DC, Benza RL. Current outcomes following heart transplantation. Semin Thorac Cardiovasc Surg. 2004;16:395-403.

4. Portner PM, Oyer PE, Pennington DG, et al. Implantable electrical left ventricular assist system: bridge to transplantation and the future. Ann Thorasc Surg. 1989;47:142-150.

5. Phillips WS, Burton NA, Macmanus Q, Lefrak EA. Surgical complications in bridging to transplantation: the Thermo Cardiosystems LVAD. Ann Thorac Surg. 1992;53:482-486.

6. Chatterjee S, Williams NN, Ohara NL, Twomey C, Morris JB, Acker MA. Diaphragmatic hernias associated with ventricular assist devices and heart transplantation. Ann Thorac Surg. 2004; 77:2111-2114.

7. Mouly-Bandini A, Chalvignac V, Collart F, et al. Transdiaphragmatic hernia 1 year after heart transplantation following implantable LVAD. J Heart Lung Transplant. 2002;21:1144-1146.

8. Nakamura T, Kohmoto T, Kao WG, Osaki S, Block KL, Weigel TL. Unusual late presentation of asymptomatic diaphragmatic hernia following ventricular assist device explantation. Interact Cardiovac Thorac Surg. 2008;7:141-143.

9. Farma J, Leeser D, Furukawa S, Dempsey DT. Laparoscopic repair of diaphragmatic hernia after left ventricular assist device. J Laparoendosc Adv Surg Tech A. 2003;13:185-187.

10. Groth SS, Whitson BA, D'Cunha J, Andrade R, Maddaus M. Diaphragmatic hernias after sequential left ventricular assist device explantation and orthotopic heart transplant: early results of laparoscopic repair with polytetrafluoroethylene. J Thorac Cardiovasc Surg. 2008;135:38-43.

11. Campos LI, Sipes EK. Laparoscopic repair of diaphragmatic hernia. J Laparoendosc Surg. 1991;1:369-373.

12. Matthews BD, Bui H, Harold KL, et al. Laparoscopic repair of traumatic diaphragmatic injuries. Surg Endosc. 2003;17:254-258.

13. Matz A, Landau O, Alis M. Charuzi I, Kyzer S. The role of laparoscopy in the diagnosis and treatment of traumatic diaphragmatic hernia. J Laparoendosc Adv Surg Tech A. 2000;8:225-299.

14. Wadhwa A, Surendra JBK, Sharma A, et al. Laparoscopic repair of diaphragmatic hernias: experience of six cases. Asian J Surg. 2005;28:145-150. 